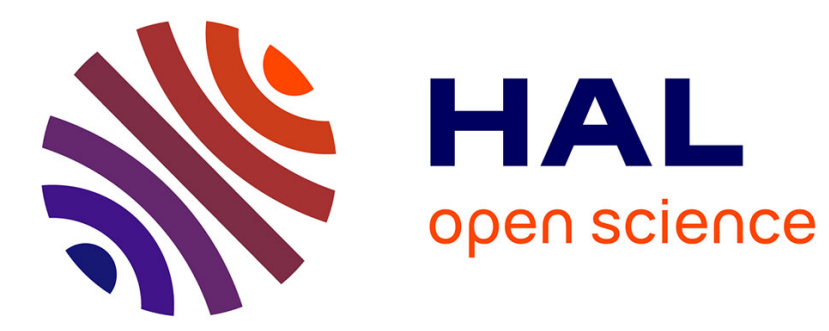

\title{
OSIrIS: a physically based simulation tool to improve training in thermal infrared remote sensing over urban areas at high spatial resolution
}

Thierry Poglio, Sandrine Mathieu-Marni, Thierry Ranchin, Eric Savaria, Lucien Wald

\section{To cite this version:}

Thierry Poglio, Sandrine Mathieu-Marni, Thierry Ranchin, Eric Savaria, Lucien Wald. OSIrIS: a physically based simulation tool to improve training in thermal infrared remote sensing over urban areas at high spatial resolution. Remote Sensing of Environment, 2006, 104, pp.238-246. 10.1016/j.rse.2006.03.017 . hal-00361375

\section{HAL Id: hal-00361375 \\ https://hal.science/hal-00361375}

Submitted on 13 Feb 2009

HAL is a multi-disciplinary open access archive for the deposit and dissemination of scientific research documents, whether they are published or not. The documents may come from teaching and research institutions in France or abroad, or from public or private research centers.
L'archive ouverte pluridisciplinaire HAL, est destinée au dépôt et à la diffusion de documents scientifiques de niveau recherche, publiés ou non, émanant des établissements d'enseignement et de recherche français ou étrangers, des laboratoires publics ou privés. 


\section{OSIrIS: a physically based simulation tool to improve training in thermal infrared remote sensing over urban areas at high spatial resolution}

Thierry Poglio ${ }^{1}$, Sandrine Mathieu-Marni ${ }^{2}$, Thierry Ranchin ${ }^{1}$, Eric Savaria ${ }^{2}$, Lucien

$$
\text { Wald }^{* 1}
$$

1- Centre Energétique et Procédés, Ecole des Mines de Paris, F-06904 Sophia Antipolis, France

2- $\quad$ Alcatel Space, System Architecture Division, F-06156 Cannes, France.

\section{Abstract}

This paper describes an infrared image simulator for remote sensing applications, called OSIrIS (outdoor scene and infrared image simulation). It has been developed partly for training and reproduces with great details the physical phenomena that play a major role in complex urban environment. OSIrIS performs a synthesis of scene based on a 3-D description of the landscape with a high spatial resolution $(0.5-10 \mathrm{~m})$. The physical processes are briefly described and their importance with respect to the objectives are discussed. Thermal emission depends on temperature and generally dominates the signal. Temperature is governed by heat equation and is solved by the means of boundary conditions such as in-depth temperature and flux balance at surface. Main parameters are solar and atmospheric radiations, wind, heat conduction and changes in humidity. An innovative approach was developed to take into account variations in time of the interactions between the landscape and the physical processes. OSIrIS aims at simulating situations that are encountered in reality. It enables users self-formation, helping them understanding changes in image radiance as a function of the input parameters and their own simulation requirements. Examples are given that illustrate specific aspects of infrared images.

\footnotetext{
* Corresponding author: CEP, Ecole des Mines de Paris, F-06904 Sophia Antipolis, France, lucien.wald@ensmp.fr
} 
Poglio T., Mathieu-Marni S., Ranchin T., Savaria E., Wald L., OSIrIS: a physically based simulation tool to improve training in thermal infrared remote sensing over urban areas at high spatial resolution. Remote Sensing of Environment, 104, 238-246, 2006, doi:10.1016/j.rse.2006.03.017

\section{Introduction}

There is now a growing demand in very high spatial resolution imagery for remote sensing application over urban areas including images in the thermal infrared range from 3 to $14 \mu \mathrm{m}$. Various domains, like meteorology and thermal radiative budget, air pollution monitoring (Wald \& Baleynaud, 1999), or security information are under concern. Such an imagery is not currently available in the public domain. Several systems are planned to provide images at a high spatial resolution of a few meters or better in a near future.

In this respect, having simulations of images that will be acquired by future sensors is necessary to train end-users with future images in an operational context. This need is enhanced by the complexity in analysis of infrared images compared to visible range. The authors undertook training activities several years ago and found that only a few tools were available for that purpose. Jaloustre-Audouin (1998) and Jaloustre et al. (1997) developed a simulator of landscape in 2-D in the infrared domain, called SPIRou. Johnson et al. (1998) developed a simulator devoted to vehicles. Gastellu-Etchegorry et al. (1996) were interested in radiative budget for vegetation canopy studies; the model DART (Gastellu-Etchegorry et al. 2004) simulates any type of landscape but the modelling of the thermal processes has a limited accuracy. Barillot (2001) developed a code called Mistral that simulates an image with a 3-D description of the landscape as input but with a limited geographical extension. Yu et al. (1998) and Wang et al. (2001, 2003) developed a model for large-scale and complex urban scenes though the feasibility of the model is to be further proven as stated by the authors themselves.

We concluded in 2000 that there was no relevant training tool that was capable of reproducing with great details and accuracy the physical phenomena that play a major role in a complex urban environment. The simulator OSIrIS (outdoor scene and infrared image simulator) was developed in part to palliate this shortcoming and better answer the expectations of the trainees. It is a simulator of infrared images dedicated to remote sensing applications and benefits from the knowledge gained with the simulator SPIRou. It takes into account a 3-D description of the landscape with a high spatial resolution $(0.5-10 \mathrm{~m})$. By exploiting up-to-date published models, this simulator aims at reproducing as accurately as possible the behaviour of the landscape and 
Poglio T., Mathieu-Marni S., Ranchin T., Savaria E., Wald L., OSIrIS: a physically based simulation tool to improve training in thermal infrared remote sensing over urban areas at high spatial resolution. Remote Sensing of Environment, 104, 238-246, 2006, doi:10.1016/j.rse.2006.03.017

providing simulations close to reality (Poglio et al., 2002a). It comprises a high quality tool modelling the physical phenomena playing a role in the infrared range and allows for an exhaustive description of the scene: buildings, streets, materials, vegetation, moisture, etc. The major requirements for the design of this training simulator are possibilities to simulate various landscapes at different geographical locations at any time for different spectral bands under various meteorological conditions. Scene synthesis is one technical approach to meet such requirements and was adopted. Of particular interest are the following IR bands as they are those present in spaceborne sensors: the so-called band II $(3-5 \mu \mathrm{m})$ and band III $(8-12 \mu \mathrm{m})$.

The modelling of the physical processes is presented in the next section. The innovative modelling of objects is then detailed. Several examples of synthesised images are presented eventually; they were selected in order to illustrate difficulty in image analysis and complexity in simulation. The first example shows the differences in spatial variability between surface temperature field and radiance field at $10 \mu \mathrm{m}$. It points out the non-linearity between temperature and radiance - a fact that is not commonly admitted - and illustrates the difficulty in interpreting the image. The second example deals with the persistency of thermal effects. The accurate simulation of such effects implies a high complexity in the description and modelling of objects. The third example illustrates the difference between the bands II and III. This difference can be large during daytime and weak during nighttime.

\section{Physics}

\section{The radiance balance equation}

In thermal infrared, the flux coming from an object and seen by a sensor originates partly from reflection and partly from thermal emission by the object. In the general case, the energy equilibrium for a set of radiating objects is expressed by the following equation, independently on the wavelength $\lambda$ (Sillion \& Puech 1994):

$$
L\left(x, \theta_{v}, \varphi_{v}\right)=L_{e}\left(x, \theta_{v}, \varphi_{v}\right)+\int_{\Omega} f_{r}\left(x, \theta, \varphi_{,}, \theta_{v}, \varphi_{v}\right) L(x, \theta, \varphi) \cos \theta d \omega
$$

where 
Poglio T., Mathieu-Marni S., Ranchin T., Savaria E., Wald L., OSIrIS: a physically based simulation tool to improve training in thermal infrared remote sensing over urban areas at high spatial resolution. Remote Sensing of Environment, 104, 238-246, 2006, doi:10.1016/j.rse.2006.03.017

- $\quad L(x, \theta, \varphi), L_{e}\left(x, \theta_{v}, \varphi_{v}\right)$ and $L_{j}(x, \theta, \varphi)$ are respectively the radiance leaving the point $x$ in the viewing direction $\left(\theta_{v}, \varphi_{v}\right)$, the radiance emitted by $x$ in the same direction, and the incident radiance impinging on $x$ from direction $(\theta, \varphi)$;

- $\Omega$ is the set of directions $(\theta, \varphi)$ in the hemisphere covering the surface at $x$;

- $f_{r}\left(x, \theta, \varphi, \theta_{v}, \varphi_{v}\right)$ is the bi-directional reflectance distribution function (BRDF) describing the reflective properties at $x$ (Nicodemus et al., 1977).

Additional information on transfer in global illumination can be found in Arvo (1993). The emitted and incident radiances and the BRDF depend upon the wavelength $\lambda$. Part of the flux $L_{i}$ reaching an object is directly reflected by this object, part of this flux is absorbed and the last part propagates through the object. Materials encountered in landscape are generally opaque and the assumption "no transmission" is usually valid. Only reflection and absorption occur.

\section{Reflection}

Spectral reflected fluxes are deduced from spectral incident fluxes. In thermal infrared from 3 to $14 \mu \mathrm{m}$, there are two sources:

- The solar flux. The major part of the energy of the Sun is in the visible range. Nevertheless, the influence of the solar flux is not negligible in infrared, particularly close to $3 \mu \mathrm{m}$. This flux is anisotropic and depends upon the relative geometry between the Sun and the object.

- The atmospheric thermal emission. The atmosphere is made of particles, gases, etc. which partly absorb radiation from the Sun or the Earth. In turn, the atmosphere radiates energy as an atmospheric emitted flux. This flux is the most significant near to $10 \mu \mathrm{m}$.

In OSIrIS, we exploit the well known simulator MODTRAN to predict spectral solar incident flux and the direct and diffuse components, in combination with the ESRA analytical model (Rigollier et al., 2000). The atmospheric flux is simulated using the model of Berger (1988). 
Poglio T., Mathieu-Marni S., Ranchin T., Savaria E., Wald L., OSIrIS: a physically based simulation tool to improve training in thermal infrared remote sensing over urban areas at high spatial resolution. Remote Sensing of Environment, 104, 238-246, 2006, doi:10.1016/j.rse.2006.03.017

\section{Thermal emission}

The main difficulty in Eq. 1 is the term $L_{e}$. According to emission laws, each object is acting as a source; its emitted flux is the product of the emissivity $\mathcal{E}_{s}$ by the blackbody function $L^{b b}$ for surface temperature $T_{s}$. For lambertian emitters, it comes:

$$
L(\lambda)=\varepsilon_{S}(\lambda) L^{b b}\left(T_{S}, \lambda\right)
$$

Spectral emissivity is an intrinsic property of the object. Due to energy conservation law for opaque objects, the sum of the absorbed and reflected fluxes must be equal to the incident flux. For objects at thermal equilibrium - and having the same temperature than the surroundings Kirchoff's law implies that absorbed and emitted fluxes must be equal. Thus, emissivity can be expressed as the complement of the reflectance to unity (Korb et al. 1999; Salisbury et al. 1994). Spectral reflectance databases (ASTER, 2000) exist for several types of materials; reflectance and emissivity are computed using such databases. A database of thermal and optical properties of objects is thus created. Emissivity of objects may depend on their moisture content, e.g., dry and wet sand. OSIrIS exploits the database and does not take into account this dependency, except if it is reported in the database.

\section{Temperature prediction}

The temperature $T$ of an object is governed by the heat equation (3). Under thermodynamical conditions usually encountered in landscape, its evolution in time is expressed as the product of the thermal diffusivity $\kappa$ of the object by the Laplacian operator $\Delta$ applied to the temperature:

$$
\frac{\partial T}{\partial t}=\kappa \Delta T
$$

Because of thermal inertia, the temperature at instant $t$ depends upon the temperature at previous instant $t$ - $d t$ and can be computed iteratively. Several solutions to Eq. 3 have been published: the finite difference method (Ciarlet \& Lions 1990; Jakob 1949; Mitchell \& Griffiths 1980) and the methods proposed by Bhumralkar (1975) and Deardorff (1978). Heat equation is a second-order equation that requires two boundary conditions: the energy flux balance at surface and the in-depth temperature. 
Poglio T., Mathieu-Marni S., Ranchin T., Savaria E., Wald L., OSIrIS: a physically based simulation tool to improve training in thermal infrared remote sensing over urban areas at high spatial resolution. Remote Sensing of Environment, 104, 238-246, 2006, doi:10.1016/j.rse.2006.03.017

At surface, the conduction flux $F_{c s}$ is given by:

$$
F_{C S}\left(T_{S}, t\right)=R_{n e t}\left(T_{S}, t\right)-\left[H\left(T_{S}, t\right)+L_{l}\left(T_{S}, t\right)\right.
$$

where $R_{n e t}$ represents the radiative flux net balance, that is sum of solar and atmospheric irradiances and the negative radiative loss by the surface. In OSIrIS as in SPIRou, each of these contributions is modelled with the parameterisations of, respectively Rigollier et al. (2000), Swinbank (1963) and Olseth et al. (1994). For the sake of space, we do not reproduce the equations of these models. $H$ denotes the convective contribution due to sensible heat; it is predicted by Louis (1979). $L_{l}$ is the latent heat and is given by the model of Noilhan and Planton (1989). Knowledge of $L_{l}$ requires also knowledge of soil moisture; the in-depth humidity of soil is predicted in a way similar to temperature. Each of these physical phenomena is modelled with an accuracy depending on its relative importance in energy flux balance (Poglio et al. 2001a): in our simulation, it is not useful to have a very accurate model for a component of low contribution. Relative locations of the objects in the landscape are taken into account as they induce disturbances in a 3-D landscape (Poglio et al., 2001a).

Given an initial state, an iterative process is harnessed to compute surface change at the instant of the simulation. The interactions between physical parameters and their variations in time are computed following Johnson (1995) and Jaloustre et al. (1997). It should be mentioned that in principle, OSIrIS may take into account the small scale variability in air temperature, water vapour pressure and wind. Nevertheless, making it a reality would require an interaction with a numerical model of atmospheric turbulent flow which has not been realised. In the following, we assume horizontal homogeneity.

\section{Modelling of urban objects}

A landscape is composed of several objects such as buildings, trees, streets, rivers, etc. Each object has attributes, such as its location, materials, internal temperature etc. The surrounding of the object is another important attribute. 
Poglio T., Mathieu-Marni S., Ranchin T., Savaria E., Wald L., OSIrIS: a physically based simulation tool to improve training in thermal infrared remote sensing over urban areas at high spatial resolution. Remote Sensing of Environment, 104, 238-246, 2006, doi:10.1016/j.rse.2006.03.017

\section{3-D objects and facets}

In computer science community (Foley et al., 1996; Watt, 2000), objects are 3-D entities made of several 2-D plane facets. Contrary to the visible range scene synthesis in the infrared image requires the knowledge of the in-depth composition of each facet in order to compute surface temperature. For instance, a wall in a building is described by an internal thermal insulation layer of few centimetres and an external layer made of concrete. Thus, landscape is modelled by the means of objects, facets, and in-depth composition of each facet.

Taking into account variations in time of all the interactions differentiates the scene synthesis in the infrared range from that in the visible domain. The synthesis methods used for short wavelengths do not need to reproduce the recent history of the landscape contrary to the infrared case. In infrared range the classical radiosity approach cannot be used, except if one applies it to each voxel and each time step. An entity, called "element", was invented for OSIrIS implementation (Poglio et al 2001b); it is detailed in the next section. It permits an efficient computation of temperatures and radiative fluxes at each instant.

\section{The element-based modelling}

The element is the 3-D entity that exhibits homogeneous properties with respect to geometrical considerations, material composition, and the physical phenomena occurring at each instant. For example, one may consider a facet partly shadowed at instant $t$. The illuminated and shadowed parts of the facet are a preliminary set of elements; this set will be subsequently subdivided by taking into account the shadowing occurring at other instants.

To reduce as much as possible the number of elements, and accordingly the computational time, an element is defined as the largest entity included in a facet. Its external surface is flat and oriented (Fig. 1). The in-depth composition is made of one or several layers of primary materials. The element is homogeneous for boundary conditions, both internal and external. At internal boundary, internal temperature is the same on each point of the boundary and each time step. At external boundary, the element is homogeneous with respect to energy flux balance at each instant during the simulation process. The mesh is made of the union of elements for the whole period of simulation. 
Poglio T., Mathieu-Marni S., Ranchin T., Savaria E., Wald L., OSIrIS: a physically based simulation tool to improve training in thermal infrared remote sensing over urban areas at high spatial resolution. Remote Sensing of Environment, 104, 238-246, 2006, doi:10.1016/j.rse.2006.03.017

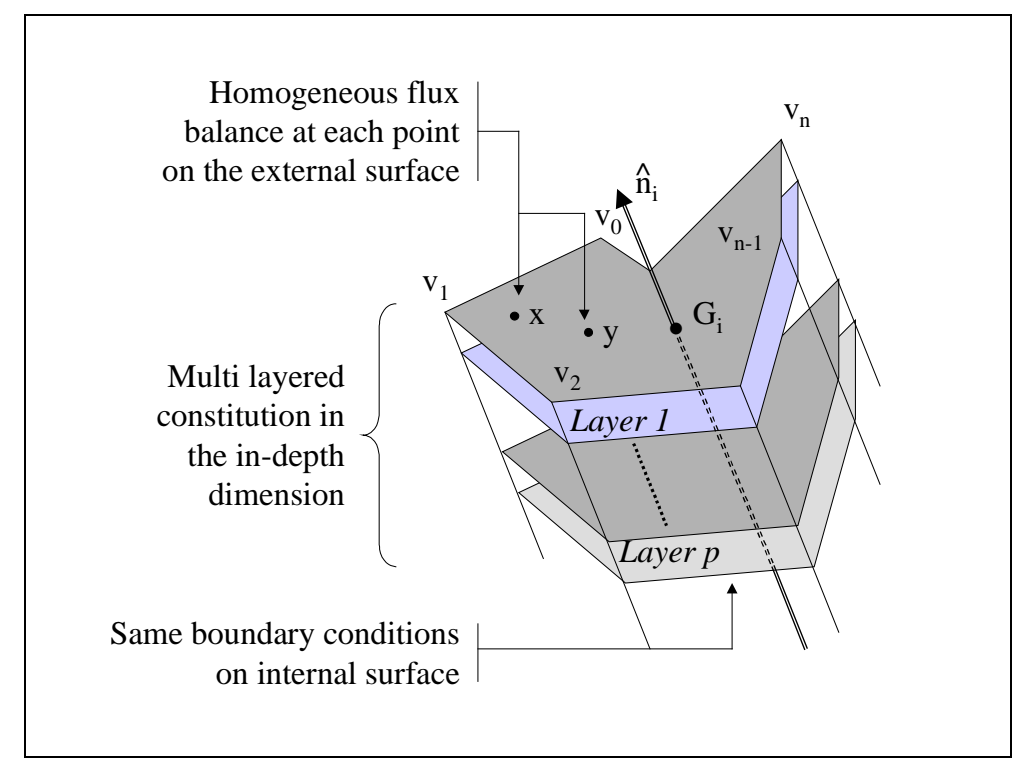

Figure 1. Illustration of the multi-layered in-depth composition of an element and the homogeneity with respect to boundary conditions on the internal and external surfaces.

The simulator OSIrIS converts the standard description of landscape by objects into an elementbased representation (Poglio et al., 2002a). Figure 2 shows the different steps used to construct the elements from the objects constituting the landscape. The element generation is detailed in Poglio et al.( 2002b). As a starting point, objects and facets representing the scene originate from the concatenation of the geometrical and material meshes. It gives rise to the initial mesh for discontinuity meshing approach (Heckbert, 1992). Identical areas with respect to boundary conditions split the scene into homogeneous areas. A new mesh taking into account these areas is achieved by the means of constrained triangulation. The latter is performed by the means of the Triangle software (Shewchuk, 1996, 2001). This new mesh is the geometrical support of the elements. Each entity of the mesh presents homogeneous properties with respect to physical phenomena at instant $t$. Shadow effects are the most important ones and sufficient accuracy can be achieved by considering this physical phenomenon only (Poglio et al., 2001a). The process is iterated to obtain the mesh at each instant during the simulation. The generation of such a mesh is based on physical considerations, which continuously change in time. There is no a priori 
Poglio T., Mathieu-Marni S., Ranchin T., Savaria E., Wald L., OSIrIS: a physically based simulation tool to improve training in thermal infrared remote sensing over urban areas at high spatial resolution. Remote Sensing of Environment, 104, 238-246, 2006, doi:10.1016/j.rse.2006.03.017

limitation to the number of meshes. Nevertheless, criteria are used to stop the refinement of the mesh according to the accuracy desired by the user (Poglio et al., 2002b).

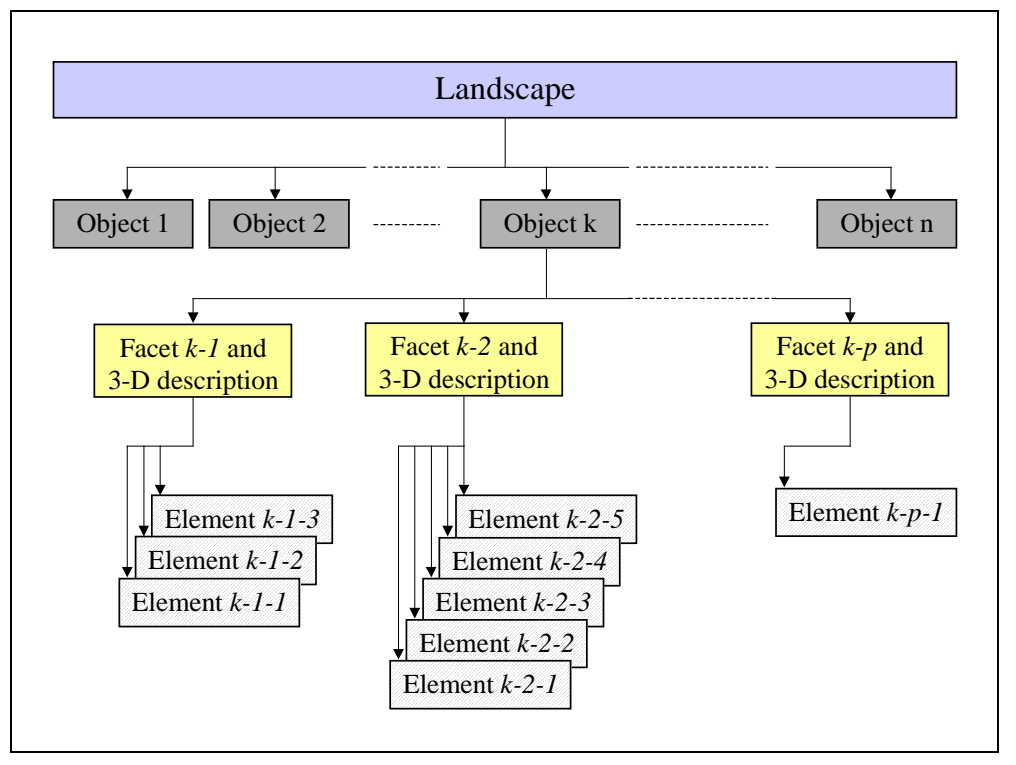

Figure 2. Illustration of the element-based representation of the landscape.

\section{Texture in thermal infrared}

In scene synthesis, texture allows to simulate the real state of the surface of a material. These textures represent the radiometric variations existing in areas assumed to be homogeneous. Textures can be considered as variations in reflection of the signal and temperature - and thus variations in thermal emission. To simulate texture, a two-steps method is used:

- firstly, the radiometry of an element is computed,

- secondly, small changes are made to the thermal and optical attributes of materials according to their domain of variation usually encountered in databases, and a second temperature is computed. The difference between this temperature and the real temperature is used to compute the amplitude of variation around the main temperature band thus around of the main radiometry. Variations of radiometry are then spatially distributed. 
Poglio T., Mathieu-Marni S., Ranchin T., Savaria E., Wald L., OSIrIS: a physically based simulation tool to improve training in thermal infrared remote sensing over urban areas at high spatial resolution. Remote Sensing of Environment, 104, 238-246, 2006, doi:10.1016/j.rse.2006.03.017

\section{Examples of use of OSIrIS for training}

\section{Temperature and radiance}

The following example shows three buildings on a flat ground (Fig. 3). In each image in this paper, North is in the upper-right corner. It means that the viewing angle is at the Southeast of the images with a tilt of $30^{\circ}$ compared to nadir viewing. The ground is made of three different materials: asphalt in the South, grass in the Northwest and sand in the Northeast; near the corner the sand is drier than in the other part. The simulation is for the $7^{\text {th }}$ of November, near the city of Cannes, South of France, 43.6 N - 7.0 E. Each facade of the three buildings is made of concrete but:

- the flat roofing of the building in the Southwest corner is covered by a thin sheet of aluminium,

- the roof of the Northern building is made of terra cotta tiles,

- the flat parts of the roof of the building in the Southeast corner are covered by aluminium and the slope roof is made of slate stone shingle.

The image on the left is an intermediate output of the OSIrIS simulator. It shows the spatial distribution of surface temperature in the 3-D scene. Surface temperatures range approximately from $3{ }^{\circ} \mathrm{C}$ in darkest black - in the shadow of the second building - to $38{ }^{\circ} \mathrm{C}$ in white - on the flat ground of the third building. The right image is a simulation in radiance in band III, between 8 and $12 \mu \mathrm{m}$, with values ranging from $26 \mathrm{Wm}^{-2} \mathrm{sr}^{-1}$ in darkest black to $42 \mathrm{Wm}^{-2} \mathrm{sr}^{-1}$ in white.

One notes that the features in these images are different. It illustrates the non-linearity between temperature and radiance, a fact not well known to trainees. One can see that the warmest surface temperature in the scene occurs on the roof of the Southwest building covered by aluminium but it does not exhibit the largest radiance. In this spectral band thermal emission dominates the signal and depends upon emissivity and surface temperature. Since aluminium has a large reflectance (Table 1), its emissivity is very low; as a consequence its thermal emission is weaker than other bodies of similar temperature but larger emissivity. Looking now carefully at the wet sand and the grass, temperatures of the sunny parts of the sand and the grass are very close whereas in the 
Poglio T., Mathieu-Marni S., Ranchin T., Savaria E., Wald L., OSIrIS: a physically based simulation tool to improve training in thermal infrared remote sensing over urban areas at high spatial resolution. Remote Sensing of Environment, 104, 238-246, 2006, doi:10.1016/j.rse.2006.03.017

shadow of the North building the corresponding temperatures are different. In the right image, these four areas exhibit four different radiances. Differences in emissivity are also responsible of these differences. 
Poglio T., Mathieu-Marni S., Ranchin T., Savaria E., Wald L., OSIrIS: a physically based simulation tool to improve training in thermal infrared remote sensing over urban areas at high spatial resolution. Remote Sensing of Environment, 104, 238-246, 2006, doi:10.1016/j.rse.2006.03.017

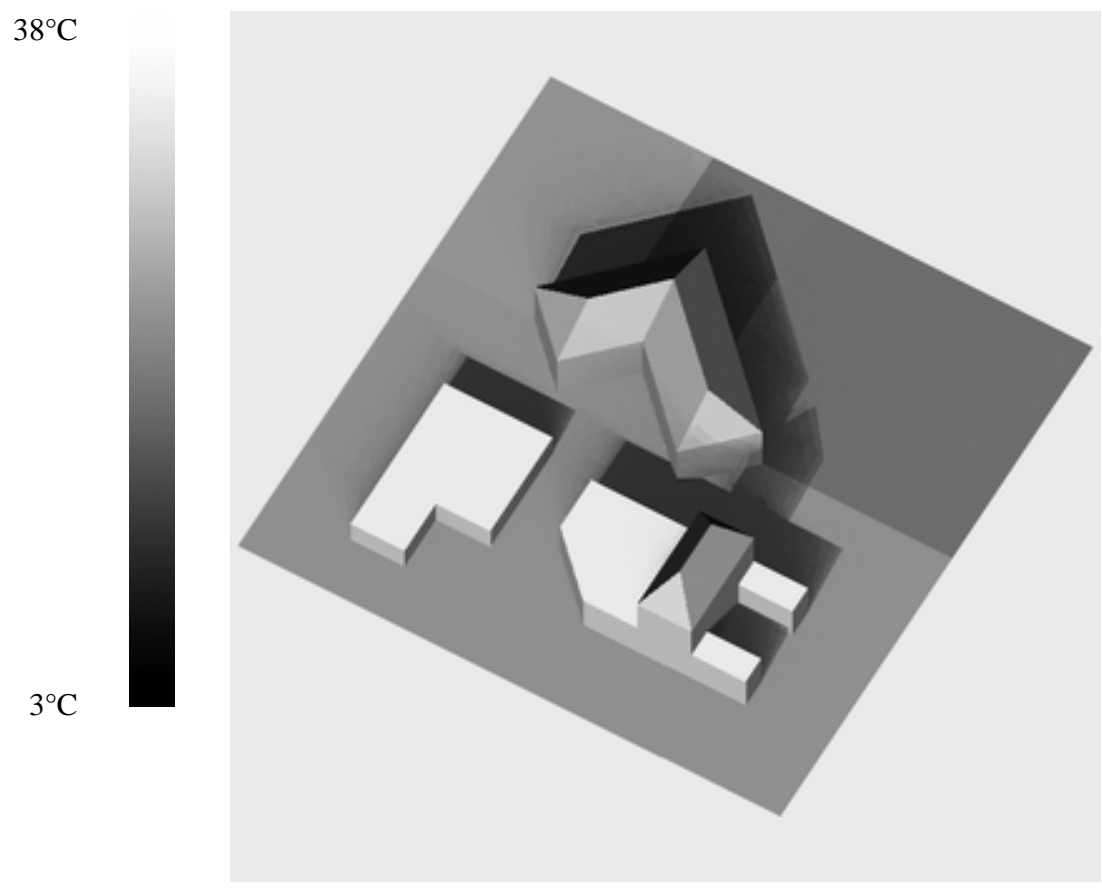

Radiance - 11:00 am - band III

$42 \mathrm{~W} \mathrm{~m}^{-2} \mathrm{st}^{-1}$

$26 \mathrm{~W} \mathrm{~m}^{-2} \mathrm{st}^{-1}$

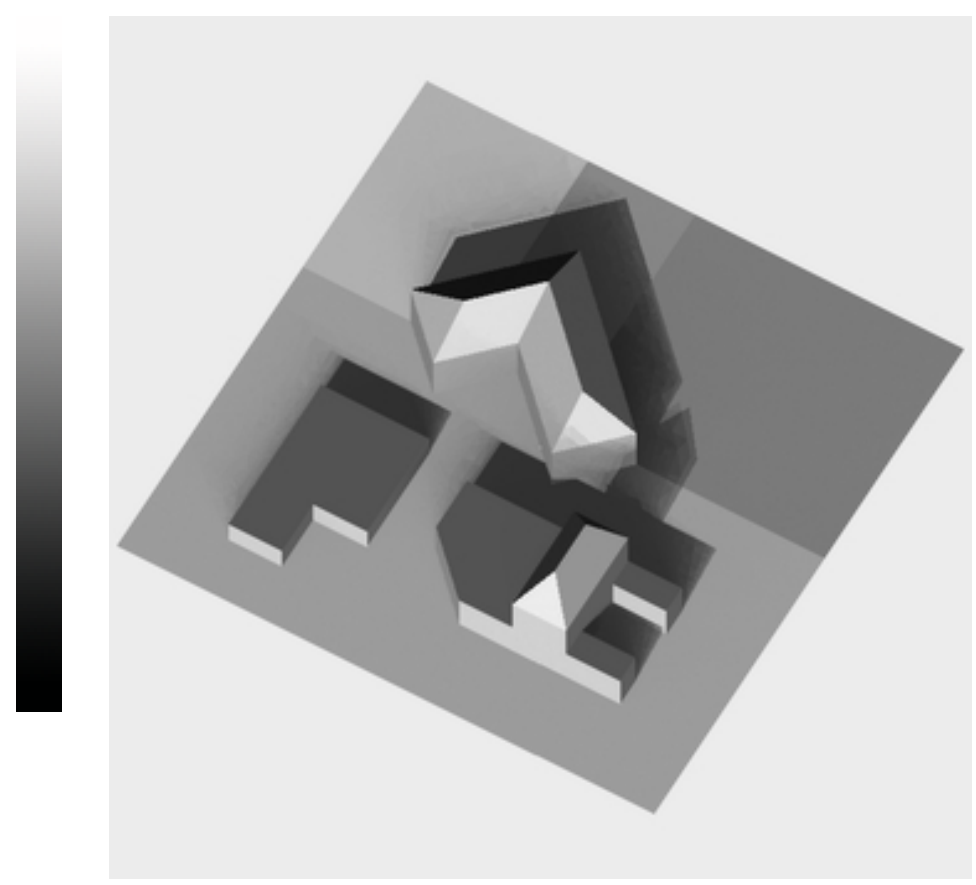

Radiance - 11:00 am - band III

Figure 3. Illustration of variations of the radiance in band II and III as a function of the temperature. 
Poglio T., Mathieu-Marni S., Ranchin T., Savaria E., Wald L., OSIrIS: a physically based simulation tool to improve training in thermal infrared remote sensing over urban areas at high spatial resolution. Remote Sensing of Environment, 104, 238-246, 2006, doi:10.1016/j.rse.2006.03.017

\section{Persistence of thermal effect}

Figure 4 shows a magnification of the central area of the previous scene for radiance in band III (Fig. 3). The radiometric profile along the measurement line is presented with distance on the $\mathrm{X}$ coordinate axis and radiances along the Y-coordinate axis. Below the distance $0 \mathrm{~m}$, values of radiance are arbitrary and should not be taken into account. In this picture, we especially focused on three areas that are typical of what is observed in infrared images. Variation along the profile is analysed from left to right.

Area \#1 is a currently sunlit area in the left of the figure. As the Sun is moving from East to West, shadows always scan the scene from West to East related to buildings location. In this case and because it is 11:00 am, this area was shadowed during the first hours of the day. Taking into account current time and scene geometry in this area, an element far from the buildings receives more solar energy than an element close to the buildings because it was not shadowed at all. Its temperature is then higher than temperature of elements near to the buildings. Reflected fluxes are the same in the whole area, and consequently the closer the element to the buildings the smaller the radiance. The continuous decrease of radiance from 35.8 to $28.4 \mathrm{Wm}^{-2} \mathrm{sr}^{-1}$ over a distance of 33 $m$ illustrates this effect of thermal persistence. In the whole image, radiance ranges from 26.3 to 42.0 $\mathrm{Wm}^{-2} \mathrm{sr}^{-1}$. Variation in this shadowed area represents more than $47 \%$ of total range of the signal. Taking into account the angle between the measurement line and the orientation of the building, and the evolution in time, this radiance variation occurs over $24 \mathrm{~m}$ from the buildings to the end of the landscape. It represents a period of $3 \mathrm{~h}$, from 8:00 to 11:00 am. Area \#3 illustrates the same phenomenon but in an opposite way. While in Area \#1 the ground is coming out of the shadow, it is going in Area \#3. The consequence is the same: the closer the pixel to the buildings, the smaller the radiance. 


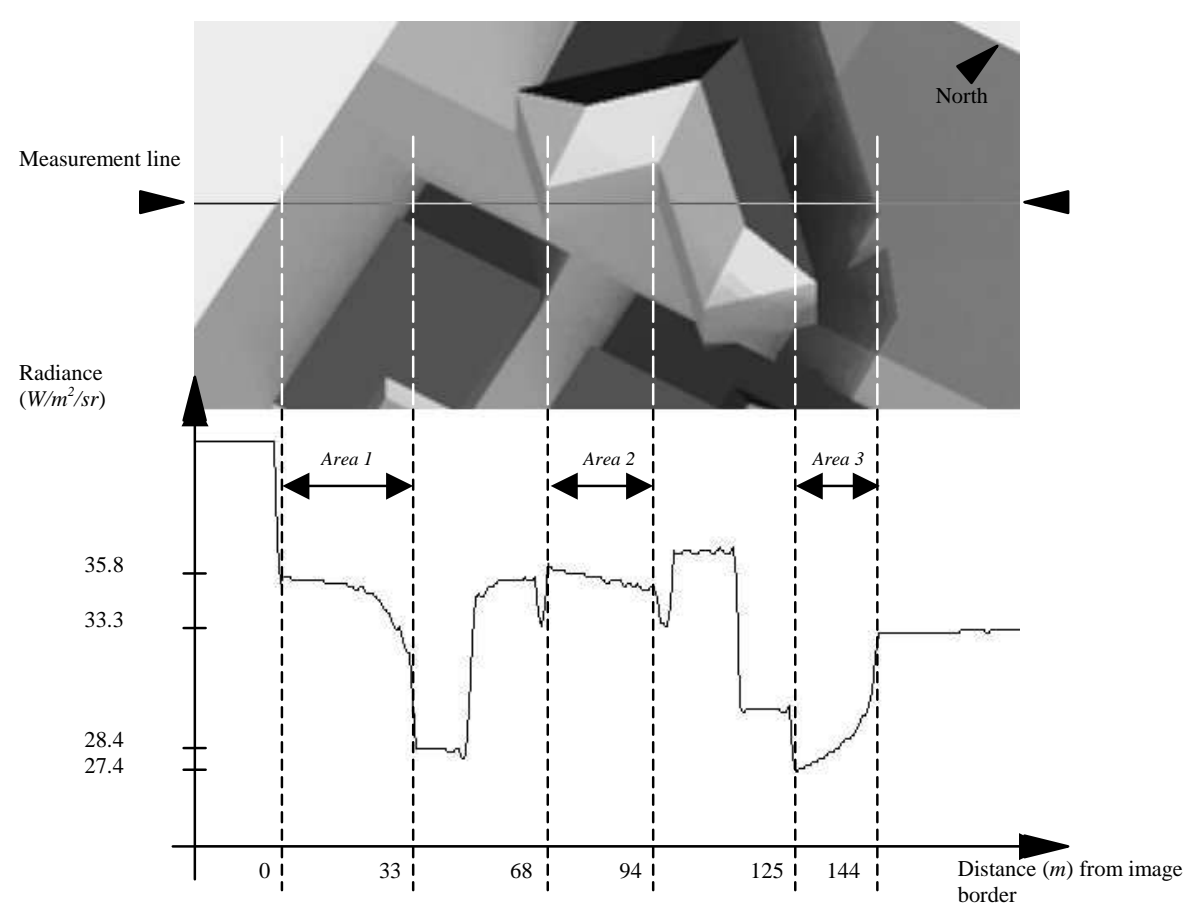

Figure 4. Radiance profile along the measurement line at 11:00 am - band III.

Area \#2 illustrates the variation of radiance as a function of the height of the pixel. This segment is on the vertical facade of the building; the height of the pixel at $68 \mathrm{~m}$ is larger than that of the pixel at $94 \mathrm{~m}$. The ground does not carry as much energy as the atmosphere to the facade and the major factor to change in temperature is the flux from the atmosphere. As the highest pixel was exposed earlier to the Sun than the lowest one, its temperature is larger. As the emissivity is homogeneous for the facade, one may observe a small increase of radiance with height.

To better illustrate the important effects of the solar shadow, a 1-D simulation is presented in Figure 5. The daily profile of the surface temperature of an object sunlit all the day long is shown in dashed line while the surface temperature of an object shadowed from sunrise to 10:00 am is in solid line. During this period, the temperature of the second (shadowed) object increases slower than the temperature of the first object. Then, when the Sun illuminates the second object, its 
Poglio T., Mathieu-Marni S., Ranchin T., Savaria E., Wald L., OSIrIS: a physically based simulation tool to improve training in thermal infrared remote sensing over urban areas at high spatial resolution. Remote Sensing of Environment, 104, 238-246, 2006, doi:10.1016/j.rse.2006.03.017

temperature increases rapidly and nearly reaches the other curve $4 \mathrm{~h}$ later. This phenomenon is called the persistence of shadow effects and has been illustrated in the previous example.

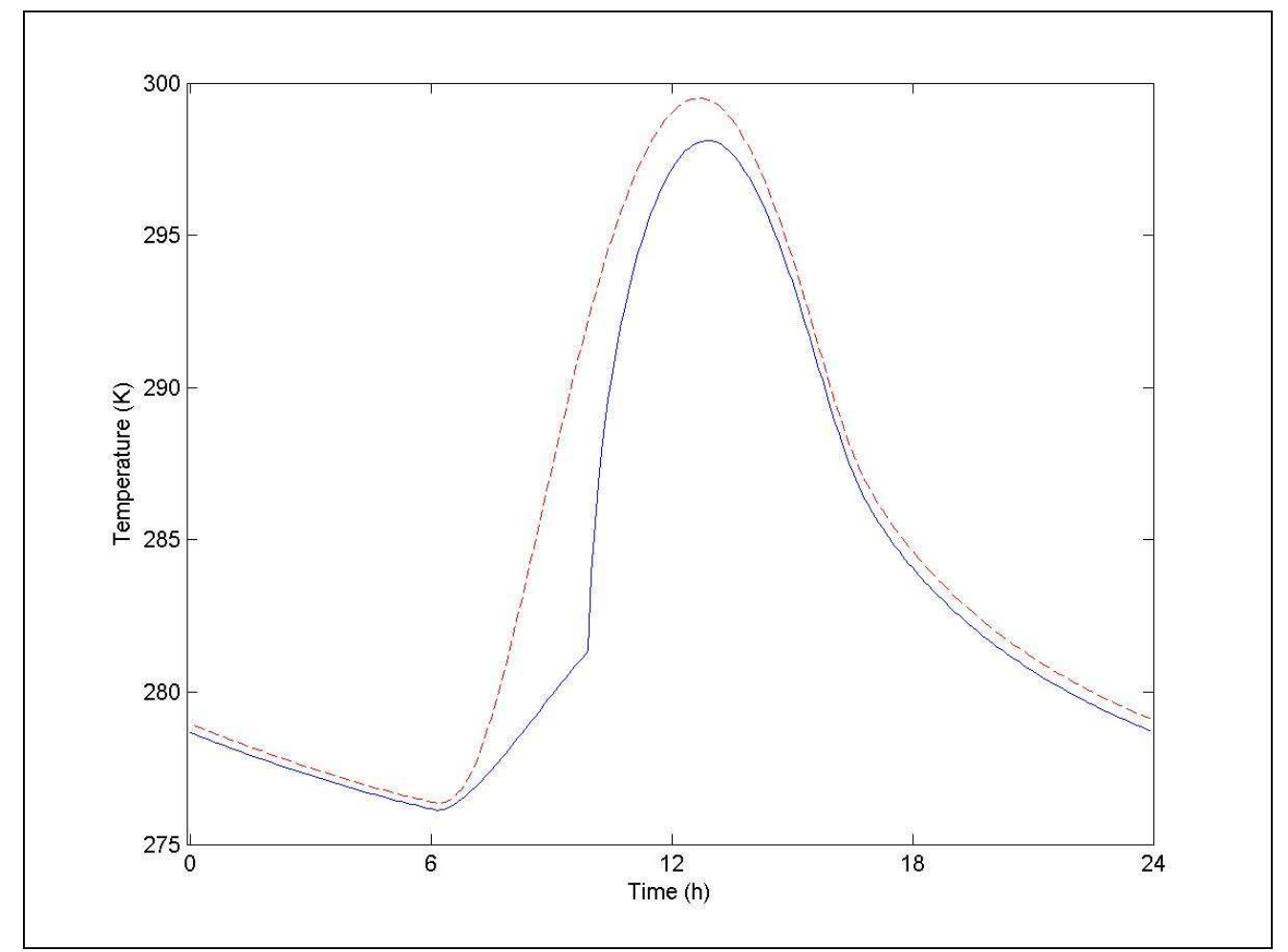

Figure 5. Daily profile of surface temperature (in Kelvin). In dashed line, temperature of an object sunlit all the day long. In solid line, temperature of an object shadowed from sunrise to 10:00 am.

\section{Impact of the spectral band}

Another interesting point for a better understanding of infrared images is the difference in behaviour between two different spectral bands. In the visible domain, the blue, green or red channels do not carry the same information. For instance, blue channel is used for ocean colour studies whereas the red one is preferred for vegetation studies. A similar situation is encountered in the infrared range, especially between band II and band III. Fig. 6 displays the spectral reflectance of four materials that were used in the previous examples: concrete and red brick for facade, terra cotta tiles and slate stone for roofing. Integrated values are reported in Table 1. Note the large value of reflectance for aluminium roofing, greater than $90 \%$ in bands II and III. These 
Poglio T., Mathieu-Marni S., Ranchin T., Savaria E., Wald L., OSIrIS: a physically based simulation tool to improve training in thermal infrared remote sensing over urban areas at high spatial resolution. Remote Sensing of Environment, 104, 238-246, 2006, doi:10.1016/j.rse.2006.03.017

values exceed what would be encountered in actual landscape; nevertheless, in a simulation context such an exaggeration helps in understanding the physical phenomena.

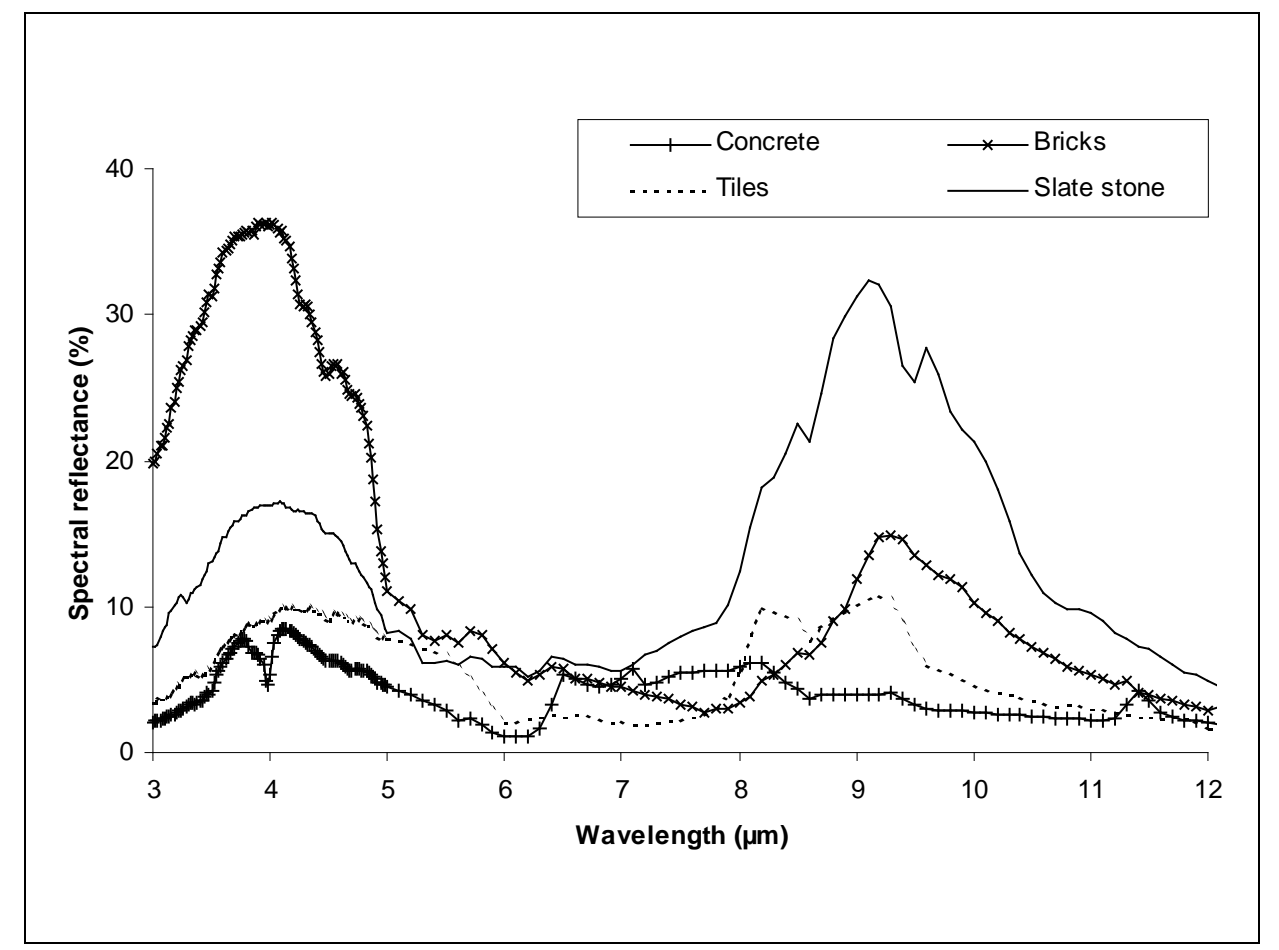

Figure 6. Spectral reflectance variations of materials used in the simulation between 3 and $12 \mu \mathrm{m}$.

\begin{tabular}{lcc}
\hline \multicolumn{1}{c}{ Materials } & Reflectance - Band II (in \%) & Reflectance - Band III (in \%) \\
\hline Front - Red smooth-faced brick & 28.5 & 7.7 \\
Front - Construction concrete & 5.6 & 3.4 \\
Roofing - Terra cotta tiles & 7.8 & 5.7 \\
Roofing - Slate stone shingle & 13.6 & 17.3 \\
Roofing - Aluminium & 91.6 & 95.0 \\
\hline
\end{tabular}

Table 1. Spectral reflectance value of materials used in the simulation for band II and band III.

Another simulation is presented in Figure 7. Fifteen buildings with six different geometries and material compositions are presented. For each building, materials are the followings:

- buildings \#1 and 4: facade made of red smooth-faced brick, roofing with terra cotta tiles, 
Poglio T., Mathieu-Marni S., Ranchin T., Savaria E., Wald L., OSIrIS: a physically based simulation tool to improve training in thermal infrared remote sensing over urban areas at high spatial resolution. Remote Sensing of Environment, 104, 238-246, 2006, doi:10.1016/j.rse.2006.03.017

- buildings \#2 and 6: facade made of concrete, roofing covered by a thin sheet of aluminium,

- building \#3: facade made of concrete, roofing with terra cotta tiles,

- building \#5: facade made of concrete, flat parts of the roof covered by aluminium, and slope roof made of slate stone shingle.

The ground is made of asphalt. Images were simulated in band II and III at 11:00 am and 9:00 pm. Because of the large variation in radiance between bands II and III, the images are encoded on 8 bits with their maximum amplitude; for each of them, the grey scale in upper left corner shows the minimum radiance value - in darkest black - and the maximum radiance value - in white. It is important to note that the radiance is always between four and ten times larger in band III than in band II. 
Poglio T., Mathieu-Marni S., Ranchin T., Savaria E., Wald L., OSIrIS: a physically based simulation tool to improve training in thermal infrared remote sensing over urban areas at high spatial resolution. Remote Sensing of Environment, 104, 238-246, 2006, doi:10.1016/j.rse.2006.03.017

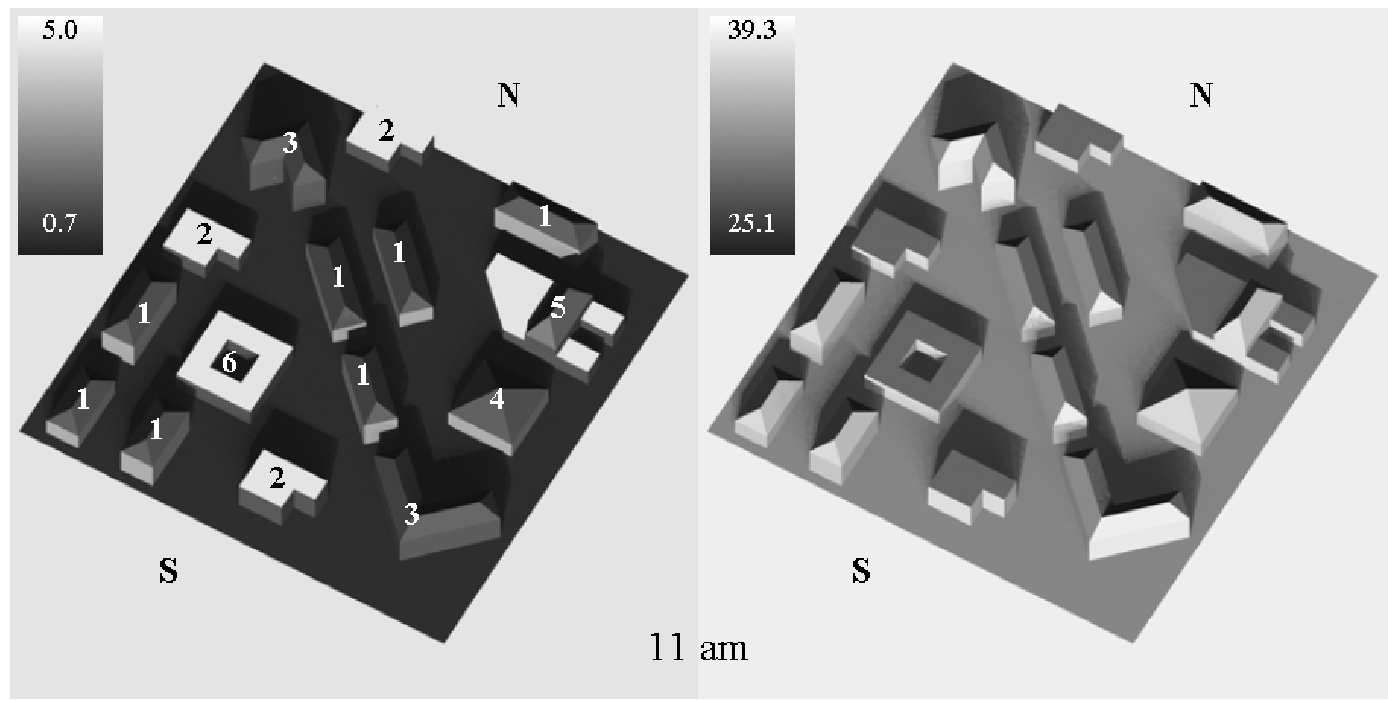

Radiance in band II - 11:00 am

Radiance in band III - 11:00 am

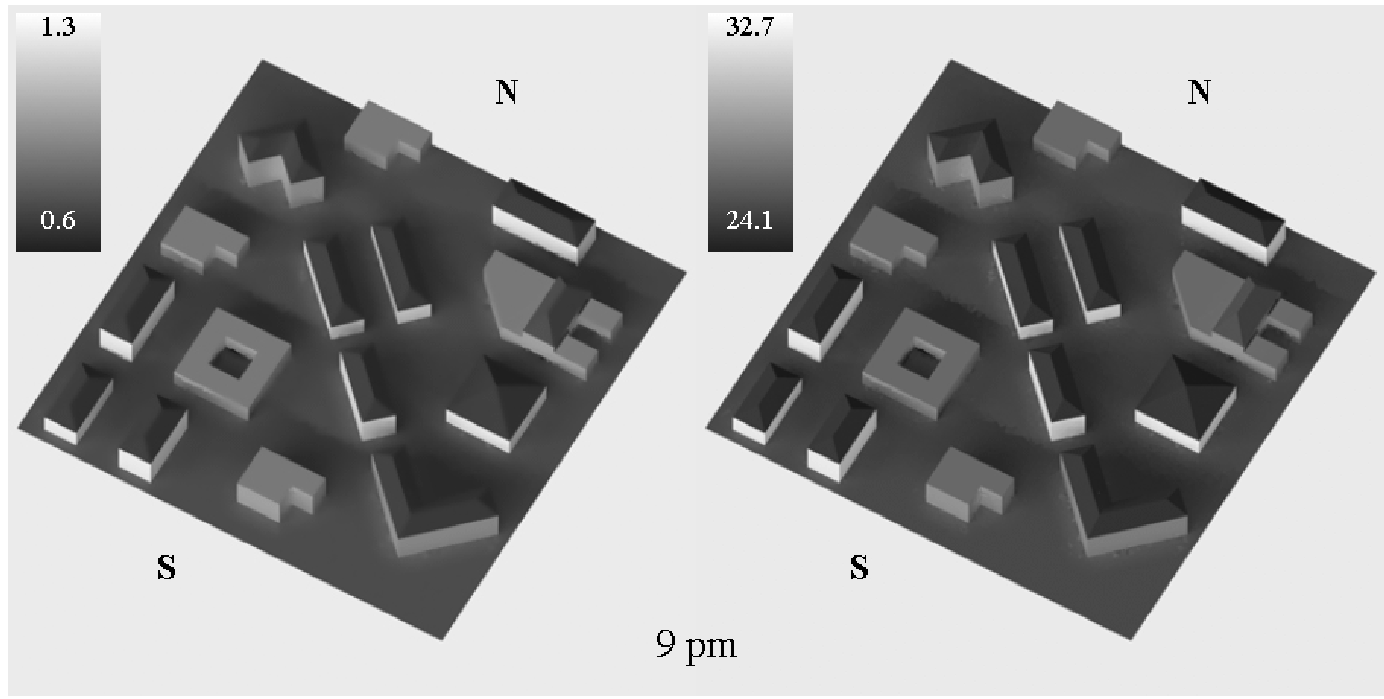

Radiance in band II - 9:00 pm

Radiance in band III - 9:00 pm

Figure 7. Four images of the same scene at 11:00 am and 9:00 pm in bands II and III. Units are $\mathrm{Wm}^{-2} \mathrm{Sr}^{-1}$

In band II - and during daytime - the scene reflects an important part of the incoming solar flux. It represents about $30 \%$ of the signal coming from the scene. The shadows of buildings appear on the ground with an important contrast compared to sunlit areas. Persistence of shadow effect is not clearly visible because it is masked by the instantaneous effect of the shadow on the reflection. In 
Poglio T., Mathieu-Marni S., Ranchin T., Savaria E., Wald L., OSIrIS: a physically based simulation tool to improve training in thermal infrared remote sensing over urban areas at high spatial resolution. Remote Sensing of Environment, 104, 238-246, 2006, doi:10.1016/j.rse.2006.03.017

shadowed areas, thermal effects of shade are less than $250 \mathrm{mWm}^{-2} \mathrm{sr}^{-1}$ : the contrast in radiance is less than $9 \%$ of the maximum radiance.

On the contrary, these thermal persistency effects clearly appear in band III, during daytime and also during night. In addition, the multi-reflection phenomenon can be observed in this band especially during night: radiance is greater near the corner of the building \#3 than on the other parts of the facade. This happens in other parts of the image.

Another interesting phenomenon is highlighted in this simulation. At night, contrasts in bands II and III appear similar. This is not exactly true because of differences in emissivity between both bands for the same material. Looking carefully at the roof of buildings 4 and 5 (terra cotta tiles and slate stone shingle), one may note a difference of contrast between radiance in bands II and III that is due to different emissivities (Table 1). The ratio of band II over band III is larger for terra cotta tiles than for slate stone shingle. Because these roofs appear with the same radiance in band II, it means that the roof of building 4 is warmer than that of building 5. Combined to different reflectance values in band III, this effect is clearly visible: radiance of building 5 is $10 \%$ larger than that of building 4 . This example illustrates the complementarities of the two spectral bands and the great difficulty of interpreting such images.

\section{Conclusion}

A simulator of realistic images should reproduce faithfully physical interactions between objects to be of high interest in training. The validation and assessment of accuracy of the outgoing radiances are a problem for such a simulator. The models used in OSIrIS have been assessed by their respective authors by comparison with in situ data and results were published. These models are combined to produce temperature prediction in a way similar to that of the simulator SPIRou. The comparisons between simulations performed with OSIrIS and SPIRou of known quality are very good (Poglio, 2002) taking into account that SPIRou is limited to a 2-D description of the landscape and a resolution of $2 \mathrm{~m}$ (Jaloustre-Audoin, 1998; Jaloustre et al. 1997). Nevertheless, the whole simulator OSIrIS has not been extensively assessed yet. The large number of parameters to input to the simulator is an obstacle to the validation. Such a validation would request a very 
Poglio T., Mathieu-Marni S., Ranchin T., Savaria E., Wald L., OSIrIS: a physically based simulation tool to improve training in thermal infrared remote sensing over urban areas at high spatial resolution. Remote Sensing of Environment, 104, 238-246, 2006, doi:10.1016/j.rse.2006.03.017

extensive and expensive campaign of measurements with well calibrated and accurate sensors: meteorology, moisture content and objects properties should be measured with great care. A comparison between OSIrIS outcomes and real infrared images of high spatial resolution shows that OSIrIS is capable of reproducing features in radiances and in gradient. What remains to be assessed is the exact accuracy of these synthesized features.

OSIrIS is helpful to users as it allows visualizing changes in radiance as a function of changes in input parameters. This capability is important for training, education and courses, including users self-training. By playing with input parameters and using their own simulation requirements (scene, dates, etc.), trainees can obtain various images that help them to better understand changes in image radiance.

The first lesson learnt in these past years is that training in infrared images is mandatory. Analysis of such images is not obvious and not as natural as it can be for images in the visible range. The second lesson is that an accurate simulator is necessary. The major physical phenomena should be reproduced with a very good accuracy, leading to images close to reality. More exactly, it is important to reproduce the heterogeneities that are generated by the complex structure of the urban fabrics, especially at high spatial resolution (for example, multiple reflections, multiple masking, complex shadows) or that are due to the variability of the meteorological fields induced by this geometric complexity, mostly wind and humidity fields.

The OSIrIS simulator and similar tools have other purposes. They help designers of new spaceborne or acquisition systems:

- to study the different system concepts and select the most relevant to the mission,

- to produce images that demonstrate the future system performances,

- to analyse the impact of the specifications on the delivered image.

They are also capable of delivering images that may serve as tests to detection and inversion algorithms, such as target recognition or temperature retrieval. 
Poglio T., Mathieu-Marni S., Ranchin T., Savaria E., Wald L., OSIrIS: a physically based simulation tool to improve training in thermal infrared remote sensing over urban areas at high spatial resolution. Remote Sensing of Environment, 104, 238-246, 2006, doi:10.1016/j.rse.2006.03.017

\section{Acknowledgements}

This research was performed under a grant of ANRT, Ministry of Research in France. The authors thank J.R. Shewchuk for the use of the softwares Triangle, the 2-D quality mesh generator and Delaunay triangulator. They also thank the anonymous referees and the Editor for their help in improving the clarity of this paper.

\section{References}

Arvo, J. (1993). Transfer equation in global illumination, Global illumination, SIGGRAPH'93 Course Notes, 42.

ASTER, (2000). ASTER spectral library Ver 1.2, CD-ROM, Jet Propulsion Laboratory, NASA, October 2000, http://spectib.jpl.nasa.gov/archive/jhu.html.

Barillot, P. (2001). MISTRAL, June 2001, http://www.onera.fr/dota/mistral/index.html.

Berger, X. (1988). A simple model for computing the spectral radiance of clear skies, Solar Energy, 40, (4), 321-333.

Bhumralkar, C.M. (1975). Numerical experiments on the computation of ground surface temperature in an atmospheric general circulation model, Journal of Applied Meteorology, 14, $1246-1258$

Ciarlet, P.G., Lions, J.L. (1990). Handbook of Numerical Analysis, Finite Difference Method (Part 1), Solution of Equations in $R^{n}$ (Part 1), Elsevier Science Publisher B.V., ISBN 0-444-70366-7, $652 \mathrm{p}$.

Deardorff, J.W. (1978). Efficient prediction of ground surface temperature and moisture with inclusion of a layer of vegetation. Journal of Geophysical Research, 83, C4, 1889-1902.

Foley, J.D., van Dam, A., Feiner, S. K., Hughes, J. F. (1996). Computer Graphics, Principles and Practice. Second Edition in C. Addison-Wesley Publishing Company, ISBN 0-201-84840-b, USA. Gastellu-Etchegorry, J.-P., Demarez, V., Pinel, V., Zagolski, F. (1996). Modeling radiative transfer in heterogeneous 3-D vegetation canopies, Remote Sensing of Environment, 58, 131-156. 
Poglio T., Mathieu-Marni S., Ranchin T., Savaria E., Wald L., OSIrIS: a physically based simulation tool to improve training in thermal infrared remote sensing over urban areas at high spatial resolution. Remote Sensing of Environment, 104, 238-246, 2006, doi:10.1016/j.rse.2006.03.017

Gastellu-Etchegorry, J.-P., Martin, E., Gascon, F. (2004). DART: a 3D model for simulating satellite images and studying surface radiation budget, International Journal of Remote Sensing, 25, (2), 73-96.

Heckbert, P.S. (1992). Discontinuity meshing for radiosity, In Proceedings of the Third Eurographics Workshop on Rendering, 203-216.

Jakob, M. (1949). Heat Transfer. Wiley, USA, 1410 p.

Jaloustre-Audoin, K. (1998). SPIRou: Synthèse de Paysage en InfraRouge par modélisation physique des échanges à la surface, Thèse de Doctorat, Université de Nice-Sophia Antipolis, Nice, France, 169 p.

Jaloustre, K., Savaria, E., Wald, L. (1997). Synthesized outdoor scenes in infrared spectral band. In Proceedings of the EARSeL Symposium, Malta.

Johnson, K.R., Wood, S.B., Rynes, P.L., Yee, B.K., Burroughs, F.C., Byrd, T. (1995). A methodology for rapid calculation of computational thermal models, SAE International Congress \& Exposition, Underhood Thermal Management Session, Detroit, MI, USA.

Johnson, K., Curran, A., Less, D., Levanen, D., Marttila, E., Gonda, T., Jones, J. (1998). MuSES: a new heat and signature management design tool for virtual prototyping, In Proceedings of the $9^{\text {th }}$ Annual Ground Target Modelling \& Validation Conference, Houghtoon, MI, USA.

Korb, A. R., Salisbury, J.W., D’Aria, D.M. (1999). Thermal-infrared remote sensing and Kirchoff's law. 2. Fields measurements. Journal of Geophysical Research, 104, 15,339-15,350.

Louis, J.-F. (1979). A parametric model of vertical eddy fluxes in the atmosphere. Boundary Layer Meteorology, 17, 187-202.

Mitchell, A.R., \& Griffiths, D.F. (1980). The Finite Difference Method in Partial Differential Equations, Wiley Intersciences Publications, ISBN 0-471-27641-3, 272 p.

Nicodemus, F.E., Richmond, J.C., Hsia, J.J. (1977). Geometrical considerations and nomenclature for reflectance, NBS Monograph 160, U.S. National Bureau of Standards, Washington, D.C, USA. Noilhan, J., Planton, S. (1989). A simple parametrization of land surface processes for meteorological models. Monthly Weather Review, 117, 536-549. 
Poglio T., Mathieu-Marni S., Ranchin T., Savaria E., Wald L., OSIrIS: a physically based simulation tool to improve training in thermal infrared remote sensing over urban areas at high spatial resolution. Remote Sensing of Environment, 104, 238-246, 2006, doi:10.1016/j.rse.2006.03.017

Olseth, A.J., Skartveit, A., Czeplak, G., Rommel, M. (1994). Review and test of parametrizations of atmospheric radiation. A technical report of task 17: Measuring and modelling spectral radiation affecting solar systems and buildings. December 1994, International Energy Agency, Wien, Austria, Report n ${ }^{\circ}$ IEA SHCP 17F 2, 124 p.

Poglio, T. (2002). Synthèse de paysage dans l'infrarouge thermique pour la simulation d'images de télédétection. Thèse de Doctorat "Sciences de l'Univers", Université de Nice-Sophia Antipolis, Nice, France, 7 novembre 2002, 126 p.

Poglio, T., Savaria, E., Wald, L. (2001a). Influence of the three-dimensional effects on the simulation of landscapes in thermal infrared. In Proceedings of the 21th EARSeL Symposium, Observing our environment from space: new solutions for a new millenium, Marne-la-Vallée, France, 14-16 May 2001, Gérard Begni editor, A. A. Balkema,, Lisse, Abingdon, Exton (PA), Tokyo, pp. 133-139.

Poglio, T., Ranchin, T., Savaria, E., Wald, L. (2001b). Simulation d'images dans l'infrarouge thermique par une approche synthétique : spécifications et architecture fonctionnelle. In ComptesRendu Journée thématique "Coopération Analyse d'Image et Modélisation, 14 juin 2001, LIGIM, Université Claude Bernard Lyon 1, Lyon, France, pp. 58-61.

Poglio, T., Savaria, E., Wald, L. (2002a). OSIRIS: a simulator of outdoor scenes in thermal infrared range, In Proceedings of the Photogrammetric Computer Vision PCV'02, ISPRS Commission III, ISSN 1682-1750, Graz, Austria, A, 240-245.

Poglio, T., Savaria, E., Wald, L. (2002b). Outdoor scene synthesis in the infrared range for remote sensing applications, In Proceedings of the International Conference on Imaging Science, Systems, and Technology, Las Vegas, USA, ISBN 1-892512-93-9, 1, 206-212.

Rigollier, C., Bauer, O., Wald, L. (2000). On the clear sky model of the ESRA - European Solar Radiation Atlas - with respect to the Heliosat method. Solar Energy, 68, (1), 33-48.

Salisbury, J.W., Wald, A., D’Aria, D.M. (1994). Thermal-infrared remote sensing and Kirchoff's law. 1. Laboratory measurements. Journal of Geophysical Research, 99, 11,897-11,911. 
Poglio T., Mathieu-Marni S., Ranchin T., Savaria E., Wald L., OSIrIS: a physically based simulation tool to improve training in thermal infrared remote sensing over urban areas at high spatial resolution. Remote Sensing of Environment, 104, 238-246, 2006, doi:10.1016/j.rse.2006.03.017

Shewchuk, J.R. (1996). Triangle: Engineering a 2D Quality Mesh Generator and Delaunay Triangulator, First ACM Whorshop on Applied Computational Geometry, Philadelphia, Pennsylvania, USA, 124-133.

Shewchuk, J.R. (2001). Triangle. A two-dimensional quality mesh generator and Delaunay triangulator. Version 1.3, Octobre 2001, http://www.cs.cmu.edu/ quake/triangle.research.html.

Sillion, F.X., \& Puech, C. (1994). Radiosity \& Global Illumination. Morgan Kaufmann Publishers, Inc., ISBN 1-558-60277-1, San Francisco, CA, U.S.A., 251 p.

Swinbank, W.C. (1963). Longwave radiation from clear skies, Q. J. R. Meteorological Research, 89, 339-348.

Wald, L., Baleynaud, J.-M. (1999). Observing air quality over the city of Nantes by means of Landsat thermal infrared data, International Journal of Remote Sensing, 20, (5), 947-959.

Wang, Z., Jiang, Z., Yu, W., Peng, Q. (2003). A realistic image synthesis model for infrared scene, International Journal of Infrared and Millimeter Waves, 24, (7), 1149-1160.

Wang, Z., Peng, Q., Lu, Y., Jiang, Z. (2001). A global infrared image synthesis model for largescale complex urban scene, International Journal of Infrared and Millimeter Waves, 22, (8), 11931208.

Watt, A. H. (2000). 3-D Computer Graphics, Third Edition, Addison-Wesley Publishing Compagny Inc, ISBN 0-201-39855-9.

Yu, W., Peng, Q., Tu, H., Wang, Z. (1998). An infrared image synthesis model based on infrared physics and heat transfer, International Journal of Infrared and Millimeter Waves, 19, (12), 16611669. 